\title{
Exponential Stability Analysis and Impulsive Tracking Control of Uncertain Time-delayed Systems
}

\author{
Yuanqiang Chen · Honglei Xu
}

Received: date / Accepted: date

\begin{abstract}
In this paper, we study exponential stability and tracking control problems for uncertain time-delayed systems. First, sufficient conditions of exponential stability for a class of uncertain time-delayed systems are established by employing Lyapunov functional methods and algebraic matrix inequality techniques. Furthermore, tracking control problems are investigated in which an uncertain linear time-delayed system is used to track the reference system. Sufficient conditions for solvability of tracking control problems are obtained for the cases that the system state is measurable and nonmeasurable, respectively. When the state is measurable, we design an impulsive control law to achieve the tracking performance. When the state information is not directly available from measurement, an impulsive control law based on the measured output will be used. Finally, numerical examples are presented to illustrate the effectiveness and usefulness of our results.
\end{abstract}

Keywords Exponential stability · Time delays · Lyapunov functional · Impulsive control $\cdot$ State tracking

\section{Introduction}

In recent decades great concerns have been addressed to stability problems of timedelayed systems since time delays are often encountered in a variety of practical systems

This work was partially supported by the National Natural Science Foundation of China under Grant 60704003, the Australian Research Council Discovery Projects, the Natural Science Foundation of Guizhou Province under Grant [2008]2252 and the Talents Foundation of Guizhou University under Grant 2007043.

\section{Yuanqiang Chen}

Department of Mathematics, Guizhou University, Guiyang 550025, China and Department of Mathematics, National Minorities College of Guizhou, Guiyang, Guizhou, 550025, China.

Honglei Xu (Corresponding author)

Department of Mathematics, Guizhou University, Guiyang 550025, China and Department of Mathematics and Statistics, Curtin University of Technology, Australia.

Tel.: +618-92664961 Fax: +618-92663197

E-mail: H. Xu@curtin.edu.au 
such as chemical processes, communication systems and power systems, etc. In particular, for neural networks, time-delayed control problems have received more attention (see, for examples, [1]-[4] and their references in which time delays can be constant or time-varying and stability criteria can be delay-dependent or delay-independent). Time-delayed systems can model these systems with interconnections between dynamics and propagation or transport phenomena. Time delays sometimes cause undesirable dynamical behaviors such as oscillation and instability. Consequently, many research results on stability criteria for time-delayed systems, such as [5]-[10], [14], have appeared in the literature. However, exponential stability problems for control systems with multiple delays and parameter uncertainty are not fully investigated and need to be developed further. This motivates us to find new stability criteria for time-delayed systems with multiple delays. In this paper, we study exponential stability problems for uncertain time-delayed systems by Lyapunov functional methods and algebraic matrix inequalities and obtain sufficient conditions for exponential stability of these systems.

On the other hand, in recent years tracking control problems have been investigated extensively, see [11]-[13] and the references therein. Time delays affect the tracking signal's transmission and implementation. To solve tracking control problems with time delays, some results have been reported in the literature, for example, see [21]-[22] and the references therein. However, these tracking control methods require that the control law should be continuous which may not be practical for some real world control systems. It is well known that the impulsive control method [16]-[20] is one of important control techniques and impulsive control laws have fast response time, low energy consumption, good robustness and resistance to disturbances. In this paper, combining with Lyapunov functional methods and algebraic matrix inequality techniques, we apply the impulsive control method to solve tracking control problems for the cases that the system state is measurable and non-measurable, respectively. Finally, the effectiveness and usefulness of our results are demonstrated by numerical examples.

Notations: Let $P>0(\geq,<, \leq)$ denote a positive definite (semi-positive definite, negative definite, semi-negative definite) matrix $P$. Let $R^{n}$ denote the $n$-dimensional Euclidean space. $\|$.$\| denotes the Euclidean norm in R^{n}$, i.e., for $x=\left[x_{1}, \ldots, x_{n}\right]^{T} \in$ $R^{n},\|x\|=\left(\sum_{l=1}^{n} x_{l}^{2}\right)^{1 / 2}$ and $\lambda_{M}(P)$ and $\lambda_{m}(P)$ are respectively the largest and the smallest eigenvalues of $P . P C\left(R_{+}, R_{+}\right)$is the set of all piecewise continuous functions $p: R_{+} \rightarrow R_{+}$such that $p \in P C\left(R_{+}, R_{+}\right)$, where $p: R_{+} \rightarrow R_{+}$is continuous on $R_{+}$ except at the time points in the set $\left\{\tau_{k}\right\}$ and is left-continuous and has right limit at $\tau_{k}$ for all $k$.

\section{Exponential stability analysis of time-delayed systems}

\subsection{System description}

Consider the following system with $k$ time delays and parameter uncertainty,

$$
\dot{x}(t)=A_{\Delta} x(t)+\sum_{i=1}^{k} B_{i \Delta}^{T} x\left(t-\tau_{i}(t)\right)+\omega(t, x),
$$

where $x(t) \in R^{n}$ is the state vector, and

$$
A_{\Delta}=A+M F N, B_{i \Delta}^{T}=\left(B_{i}+M F L_{i}\right)^{T},
$$


$F^{T} F \leq I, A, M, N, B_{i}, L_{i}(i=1,2, \cdots, k)$ are known real constant matrices with appropriate dimensions, $F$ is an uncertain real matrix, $I$ is an identity matrix, $\omega(t, x)$ is a bounded external disturbance described by a continuous vector-valued function and $\tau_{i}(t)(i=1,2, \cdots k)$ is the $i$ th differentiable time-delayed function where

$$
\begin{gathered}
x\left(t-\tau_{i}(t)\right)=\left(x_{1}\left(t-\tau_{i 1}(t)\right), x_{2}\left(t-\tau_{i 2}(t)\right), \cdots, x_{n}\left(t-\tau_{i n}(t)\right)\right)^{T}, \\
\tau^{*}=\sup _{t \geq 0}\left\{\max _{1 \leq i \leq k, 1 \leq j \leq n}\left\{\tau_{i j}(t)\right\}\right\}<\infty, \hat{\tau}=\max _{1 \leq i \leq k}\left\{\sup _{t \geq 0}\left\{\tau_{i}^{\prime}(t)\right\}\right\} .
\end{gathered}
$$

First, we need to introduce some preliminary concepts which will be used throughout this paper.

Definition 1 Assume that for system (1) there exist positive constants $r>0$ and $\mu>1$ such that

$$
\|x(t)\| \leq \mu e^{-r t} \sup _{-\tau^{*} \leq t \leq 0}\|x(t)\| .
$$

Then, the trivial solution of system (1) is said to be exponentially stable and $r$ is called the convergence rate of exponential stability.

Lemma 1 ([10]) Let $H, P$ be real matrices of appropriate dimensions. Then, for any appropriate matrix $S>0$ and any scalar $\delta>0$, the following inequality holds,

$$
H P+P^{T} H^{T} \leq \delta^{-1} H S^{-1} H^{T}+\delta P^{T} S P
$$

2.2 Stability criteria

We now present our sufficient conditions of exponential stability for system (1).

Theorem 1 Let

$$
Q_{i}=\operatorname{diag}\left(q_{11}^{(i)}, q_{22}^{(i)}, \ldots, q_{n n}^{(i)}\right)>0, \quad(i=1,2, \ldots, k)
$$

$\|\omega(t, x)\| \leq l\|x(t)\|, l \geq 0$, and $\hat{\tau}<1$. Then, system (1) is exponentially stable and the convergence rate of exponential stability is $r=\frac{|\bar{\lambda}|}{2}$ if the following inequality holds,

$G=A+A^{T}+M M^{T}+N^{T} N+\sum_{i=1}^{k} Q_{i}+\frac{2}{1-\hat{\tau}} \sum_{i=1}^{k} \frac{1}{\lambda_{i}}\left(B_{i}^{T} B_{i}+\lambda_{0} L_{i}^{T} L_{i}\right)+\left(1+l^{2}\right) I<0$,

where $\bar{\lambda}, \lambda_{0}$ are respectively the largest eigenvalues of $G$ and $M^{T} M$, and $\lambda_{i}$ is the smallest eigenvalue of $Q_{i}$.

Proof Choose a Lyapunov-Krasovskii functional candidate as

$$
V(x(t))=x^{T}(t) x(t)+\sum_{i=1}^{k} \int_{t-\tau_{i}(t)}^{t} x^{T}(s) Q_{i} x(s) d s
$$

where $Q_{i}>0,(i=1,2, \cdots, k)$. Taking the derivative of the Lyapunov functional (5) along the solution of system (1), we obtain

$$
\dot{V}(x(t))=\dot{x}^{T}(t) x(t)+x^{T}(t) \dot{x}(t)
$$




$$
\begin{gathered}
+\sum_{i=1}^{k} x^{T}(t) Q_{i} x(t)-\sum_{i=1}^{k}\left(1-\tau_{i}^{\prime}(t)\right) x^{T}\left(t-\tau_{i}(t)\right) Q_{i} x\left(t-\tau_{i}(t)\right) \\
\leq x^{T}(t)\left(A_{\Delta}^{T}+A_{\Delta}\right) x(t)+2 \sum_{i=1}^{k} x^{T}\left(t-\tau_{i}(t)\right) B_{i \Delta} x(t)+2 x^{T}(t) \omega(t) \\
+\sum_{i=1}^{k} x^{T}(t) Q_{i} x(t)-\sum_{i=1}^{k}(1-\hat{\tau}) x^{T}\left(t-\tau_{i}(t)\right) Q_{i} x\left(t-\tau_{i}(t)\right) \\
\leq x^{T}(t)\left(A^{T}+A+M F N+N^{T} F^{T} M^{T}+\sum_{i=1}^{k} Q_{i}\right) x(t)+2 \sum_{i=1}^{k} x^{T}\left(t-\tau_{i}(t)\right) B_{i \Delta} x(t) \\
+2 x^{T}(t) \omega(t)-\sum_{i=1}^{k}(1-\hat{\tau}) \lambda_{i} x^{T}\left(t-\tau_{i}(t)\right) x\left(t-\tau_{i}(t)\right)
\end{gathered}
$$

By Lemma 1, the following inequalities hold,

$$
\begin{gathered}
M F N+N^{T} F^{T} M^{T} \leq M M^{T}+N^{T} N, \\
2 x^{T}(t) \omega(t) \leq x^{T}(t) x(t)+\omega^{T}(t) \omega(t) \leq\left(1+l^{2}\right) x^{T}(t) x(t), \\
2 x^{T}\left(t-\tau_{i}(t)\right) B_{i \Delta} x(t) \leq \frac{1}{(1-\hat{\tau}) \lambda_{i}} x^{T}(t) B_{i \Delta}^{T} B_{i \Delta} x(t) \\
+(1-\hat{\tau}) \lambda_{i} x^{T}\left(t-\tau_{i}(t)\right) x\left(t-\tau_{i}(t)\right),
\end{gathered}
$$

and

$$
\begin{gathered}
B_{i \Delta}^{T} B_{i \Delta}=B_{i}^{T} B_{i}+L_{i}^{T} F^{T} M^{T} M F L_{i}+L_{i}^{T} F^{T} M^{T} B_{i}+B_{i}^{T} M F L_{i} \\
\leq 2 B_{i}^{T} B_{i}+2 \lambda_{0} L_{i}^{T} L_{i} .
\end{gathered}
$$

Then, substituting the above inequalities to (6) gives

$$
\begin{gathered}
\dot{V}(x(t)) \leq x^{T}(t)\left(A^{T}+A+M M^{T}+N^{T} N+\sum_{i=1}^{k} Q_{i}\right) x(t) \\
+\sum_{i=1}^{k} \frac{2}{(1-\hat{\tau}) \lambda_{i}}\left(B_{i}^{T} B_{i}+\lambda_{0} L_{i}^{T} L_{i}\right)+x^{T}(t)\left(1+l^{2}\right) I x(t)=x^{T}(t) G x(t) .
\end{gathered}
$$

Thus, it follows from (4) and (7) that

$$
\dot{V}(x(t))<0, \quad \forall t \geq 0 .
$$

Moreover, it is clear that there exists a positive scalar $\alpha>1$ such that the following inequalities are satisfied,

$$
\|x(t)\|^{2}<V(x(t)) \leq \alpha\|x(t)\|^{2}, \forall t \geq 0 .
$$

Thus,

$$
\|x(t)\|^{2}<V(x(t))<V(x(0)) \leq \alpha\|x(0)\|^{2}, \forall t \geq 0 .
$$


By (9), we have

$$
\begin{gathered}
\ln \frac{\|x(t)\|^{2}}{\alpha\|x(0)\|^{2}}<\ln \frac{V(x(t))}{V(x(0))} \\
=\int_{0}^{t} \frac{\dot{V}(x(s))}{V(x(s))} d s \\
<\int_{0}^{t} \frac{x(s)^{T} G x(s)}{\|x(s)\|^{2}} d s \\
\leq \int_{0}^{t} \frac{\bar{\lambda}\|x(s)\|^{2}}{\|x(s)\|^{2}} d s \\
=\bar{\lambda} t=-2 r t,
\end{gathered}
$$

where $r=\frac{|\bar{\lambda}|}{2}$. Thus, we have from (10) that

$$
\|x(t)\|<\sqrt{\alpha} e^{-r t}\|x(0)\|,
$$

which implies that

$$
\|x(t)\|<\sqrt{\alpha} e^{-r t} \sup _{-\tau^{*} \leq t \leq 0}\|x(t)\|,
$$

where $\sqrt{\alpha}>1, r>0$. Therefore, in accordance with Definition 1, system (1) is exponentially stable and the convergence rate of exponential stability is $r=\frac{|\bar{\lambda}|}{2}$.

\section{Impulsive tracking control of time-delayed systems}

3.1 System description and basic impulsive theory

We now study the following tracking control problem. For a reference linear system

$$
\begin{aligned}
& \dot{x}_{r}(t)=A_{r} x_{r}(t), \\
& x_{r}(0)=x_{r 0},
\end{aligned}
$$

we consider a tracking linear system with time delays and disturbances

$$
\begin{aligned}
& \dot{x}(t)=A x(t)+B x(t-h)+w(t), \\
& y(t)=C x(t), \\
& x(0)=x_{0},
\end{aligned}
$$

and the state tracking performance index

$$
\lim _{t \rightarrow+\infty}\left\|x_{r}(t)-x(t)\right\|=0
$$

where $x_{r}(t), x(t) \in R^{n}$ are the state vectors of the reference system and tracking system respectively, $y(t) \in R^{m}$ is the output vector, $A_{r}, A, B$ and $C$ are constant matrices of appropriate dimensions, $w(t)$ is a bounded external disturbance described by a continuous vector-valued function and $h$ is the delay time. 
We are now in a position to introduce some basic impulsive control theory. Consider the following impulsive control system with time delays,

$$
\begin{aligned}
\dot{x}(t) & =f(t, x(t), x(t-h)), t \neq \tau_{k}, \\
\Delta x(t) & =x\left(t^{+}\right)-x\left(t^{-}\right)=u_{k}(x), t=\tau_{k}, \\
x\left(\tau_{0}^{+}\right) & =x(0), k=1,2, \cdots,
\end{aligned}
$$

where $f \in C\left(R_{+} \times R^{n} \times R^{n}, R^{n}\right), u_{k} \in C\left(R^{n}, R^{n}\right)$, while $0<\tau_{1}<\cdots<\tau_{k}<\cdots$, with $\tau_{k} \rightarrow \infty$ as $k \rightarrow \infty$.

Definition 2 The state of system (12) impulsively asymptotically tracks that of system (11) if there exists an impulsive control law $\left\{\tau_{k}, u_{k}(x)\right\}$ such that $\lim _{t \rightarrow+\infty}\left\|x_{r}(t)-x(t)\right\|=$ 0 holds.

Definition 3 For each $\rho>0$, define

$$
S_{\rho}=\left\{x \in R^{n}:\|x(t)\|<\rho\right\},
$$

and for $(t, x) \in\left(\tau_{k-1}, \tau_{k}\right] \times R^{n}, k=1,2, \ldots$, let

$$
D^{+} V(t, x)=\lim _{h \rightarrow 0} \sup \frac{1}{h}[V(t+h, x+h f(t, x))-V(t, x)] .
$$

Definition 4 Let $V_{0}$ be the set containing all functions $V(\cdot, \cdot): R_{+} \times S_{\rho} \rightarrow R_{+}$which are continuous on $R_{+} \times S_{\rho}$, except possibly at a sequence of points $\left\{\tau_{k}\right\}$, and satisfy the following two conditions,

i) for each $x \in S_{\rho}, k=1,2, \ldots, \quad \lim _{(t, y) \rightarrow\left(\tau_{k}^{-}, x\right)} V(t, y)=V\left(\tau_{k}^{-}, x\right)$ exists;

ii) $V(t, x)$ is locally Lipschitz in $x$.

Moreover, the following lemma gives sufficient conditions for asymptotical stability of system (14).

Lemma 2 ([20]) Assume that there exist $\alpha, \beta, \gamma, g \in K, p \in P C\left(R_{+}, R_{+}\right), V(t, x) \in V_{0}$ and $\sigma>0$, such that the following conditions are satisfied.

i) $\beta(\|x\|) \leq V(t, x) \leq \alpha(\|x\|), \forall(t, x) \in[-h, \infty) \times S_{\rho}$;

ii) $V\left(\tau_{k}, \varphi(0)+u_{k}\left(\varphi\left(\tau_{k}\right)\right)\right) \leq g\left(V\left(\tau_{k}^{-}, \varphi(0)\right)\right), \forall\left(\tau_{k}, \varphi\right) \in R_{+} \times P C\left([-h, 0], S_{\rho}\right)$;

iii) $D^{+} V(t, \varphi(0)) \leq p(t) \gamma(V(t, \varphi(0))), \forall t \in R_{+}, t \neq \tau_{k}$ and $\varphi \in P C\left([-h, 0), S_{\rho}\right)$, when $V(t, \varphi(0)) \geq g(V(t+s, \varphi(s))), \forall s \in[-h, 0)$;

iv) $G_{2}=\inf _{q>0} \int_{g(q)}^{q} \frac{d s}{\gamma(s)}>\sup _{t \geq 0} \int_{t}^{t+\tau} p(s) d s=G_{1}$, where $\varphi\left(0^{-}\right)=\varphi(0), \tau=\sup \left\{\tau_{k}-\tau_{k-1}\right\}<$

$\infty$. Then, the trivial solution of the impulsive system (14) is asymptotically stable.

\subsection{Tracking control criteria}

\subsubsection{Measurable state case}

We first consider the case that the state of system (12) is measurable. Under the designed impulsive control law $\left\{\tau_{k}, u_{k}(x)\right\}$, where $u_{k}(x)=Q_{r} x_{r}(t)-Q x(t), Q, Q_{r} \in$ 
$R^{n \times n}$, system (12) can be rewritten as

$$
\begin{aligned}
& \dot{x}(t)=A x(t)+B x(t-h)+w(t), t \neq \tau_{k}, \\
& \Delta x(t)=Q_{r} x_{r}(t)-Q x(t), t=\tau_{k}, \\
& y(t)=C x(t), \\
& x\left(\tau_{0}^{+}\right)=x(0)=x_{0}, k=1,2, \cdots .
\end{aligned}
$$

We thus have the following theorem.

Theorem 2 Let $P>0,\left\|x_{r}(t)-x(t)\right\| \leq l\|x(t)\|,\|x(t-h)\| \leq \frac{1}{\sqrt{\eta}}\|x(t)\|$ and $\|w(t)\| \leq$ $\mu\|x(t)\|$. Then the state of system (12) impulsively asymptotically tracks that of the reference system (11) if the following inequality holds,

$$
0<\eta e^{\frac{\tau}{\eta}}<e^{-\tau\left(1+\lambda_{M}(E)+\frac{\mu^{2}}{\lambda_{m}(P)}\right)}<1
$$

where $\eta=2\left(\lambda_{M}(F)+\frac{\lambda_{M}(G)(1+l)^{2}}{\lambda_{m}(P)}\right)>0, F=I-2 Q+P^{-1} Q^{T} P Q, G=Q_{r}^{T} P Q_{r}$ and $E=A+P^{-1} A^{T} P+B P^{-1} B^{T} P$.

Proof Choose the following Lyapunov functional candidate as

$$
V(t, x)=x(t)^{T} P x(t),
$$

which clearly satisfies condition (i) of Lemma 2.

At $t=\tau_{k}, k=1,2, \ldots$, we have

$$
\begin{gathered}
V\left(\tau_{k}^{+}, x\right)=x\left(\tau_{k}^{+}\right)^{T} P x\left(\tau_{k}^{+}\right) \\
=x\left(\tau_{k}\right)^{T}\left(P-2 P Q+Q^{T} P Q\right) x\left(\tau_{k}\right)+2 x\left(\tau_{k}\right)^{T}\left(P Q_{r}-Q^{T} P Q_{r}\right) x_{r}\left(\tau_{k}\right) \\
+x_{r}\left(\tau_{k}\right)^{T} Q_{r}^{T} P Q_{r} x_{r}\left(\tau_{k}\right) \\
\leq 2 x\left(\tau_{k}\right)^{T}\left(P-2 P Q+Q^{T} P Q\right) x\left(\tau_{k}\right)+2 x_{r}\left(\tau_{k}\right)^{T} Q_{r}^{T} P Q_{r} x_{r}\left(\tau_{k}\right)^{T} \\
\leq 2\left(\lambda_{M}(F)+\frac{\lambda_{M}(G)(1+l)^{2}}{\lambda_{m}(P)}\right) V\left(\tau_{k}, x\right)=\eta V\left(\tau_{k}, x\right) .
\end{gathered}
$$

Thus, condition (ii) of Lemma 2 is satisfied with $g(s)=\eta s$. On the other hand, by virtue of the Lyapunov function (17), taking upper Dini derivative along the solution of system (15) in the time interval $\left\{t \mid t \neq \tau_{k}, k=1,2, \ldots\right\}$, we obtain

$$
\begin{gathered}
D^{+} V(t, x)=x(t)^{T}\left(A^{T} P+P A\right) x(t)+2 x(t)^{T} P B x(t-h)+2 x(t)^{T} P w(t) \\
\leq x(t)^{T}\left(A^{T} P+P A\right) x(t)+2 x(t)^{T} P w(t)+x(t)^{T} P B P^{-1} B^{T} P x(t)+\frac{1}{\eta} V(t) \\
\leq x(t)^{T}\left(A^{T} P+P A\right) x(t)+\left(1+\frac{\mu^{2}}{\lambda_{m}(P)}\right) V(t)+x(t)^{T} P B P^{-1} B^{T} P x(t)+\frac{1}{\eta} V(t) \\
\leq\left(1+\lambda_{M}(E)+\frac{1}{\eta}+\frac{\mu^{2}}{\lambda_{m}(P)}\right) V(t) .
\end{gathered}
$$

Thus, condition (iii) is satisfied with $p(t)=1+\lambda_{M}(E)+\frac{1}{\eta}+\frac{\mu^{2}}{\lambda_{m}(P)}$, and $\gamma(s)=s$. For $\varepsilon<\tau$, we have the following two integrals,

$$
G_{1}=\sup _{t \geq 0} \int_{t}^{t+\tau} p(s) d s=\left(1+\lambda_{M}(E)+\frac{1}{\eta}+\frac{\mu^{2}}{\lambda_{m}(P)}\right) \tau
$$


and

$$
G_{2}=\inf _{q>0} \int_{g(q)}^{q} \frac{d s}{\gamma(s)}=-\ln \eta .
$$

By (16), the following inequality holds,

$$
G_{2}>G_{1} .
$$

Thus, condition (iv) of Lemma 2 is also satisfied. Therefore, system (5) is asymptotically stable. In addition, we have $\lim _{t \rightarrow+\infty}\|x(t)\|=0$.

By $\left\|x_{r}(t)-x(t)\right\| \leq l\|x(t)\|$, it follows that $\lim _{t \rightarrow+\infty}\left\|x_{r}(t)-x(t)\right\|=0$. Thus, the state of system (12) impulsively asymptotically tracks that of the reference system (11).

\subsubsection{Non-measurable case}

We now turn to another case. The impulsive controller designed above is no longer effective when the state of system (12) is not directly available from measurement. We now consider a new impulsive control law based on the measured output rather than the state information.

Consider the state estimator of system (12) described by

$$
\begin{aligned}
& \dot{\bar{x}}(t)=A \bar{x}(t)+B \bar{x}(t-h)+L C(x(t)-\bar{x}(t))+w(t), \\
& \bar{y}(t)=C \bar{x}(t), \\
& \bar{x}(0)=\bar{x}_{0},
\end{aligned}
$$

where $L$ is the output feedback gain matrix. Define the difference between the real state and the estimator state as

$$
e(t)=x(t)-\bar{x}(t)
$$

From (12) and (18), we have

$$
\begin{aligned}
& \dot{e}(t)=(A-L C) e(t)+B e(t-h), \\
& y_{e}(t)=C e(t), \\
& e(0)=e_{0}
\end{aligned}
$$

Then, we have the following theorem for the asymptotical stability of the error system (19).

Theorem 3 System (19) is asymptotically stable if there exists an output feedback matrix $L$ such that the following inequality holds,

$$
J=A+A^{T}+I-L C-C^{T} L^{T}+B B^{T}<0 .
$$

Proof Choose the following Lyapunov functional candidate as

$$
V(t, e(t))=e(t)^{T} e(t)+\int_{t-h}^{t} e(s)^{T} e(s) d s .
$$


Obviously, $V(t, e(t))>0$. By virtue of the Lyapunov function $V(t, e(t))$, taking upper Dini derivative along the solution of system (19), we obtain

$$
\begin{gathered}
\dot{V}(t, e(t))=\dot{e}(t)^{T} e(t)+e(t)^{T} \dot{e}(t)+e(t)^{T} e(t)-e(t-h)^{T} e(t-h) \\
=e(t)^{T}\left(A+A^{T}+I-L C-C^{T} L^{T}\right) e(t)+2 e(t)^{T} B e(t-h)-e(t-h)^{T} e(t-h) \\
\leq e(t)^{T}\left(A+A^{T}+I-L C-C^{T} L^{T}+B B^{T}\right) e(t) .
\end{gathered}
$$

By (20), it is easy to see that

$$
\dot{V}(t, e(t))<0
$$

Thus, system (19) is asymptotically stable.

We now investigate the problems that the state of estimator system (18) asymptotically tracks that of the reference system (11). If the difference between the real state and the estimator state is regarded as the external disturbance of system (18), then (18) can be rewritten as

$$
\begin{aligned}
& \dot{\bar{x}}(t)=A \bar{x}(t)+B \bar{x}(t-h)+\bar{w}((t)), \\
& \bar{y}(t)=C \bar{x}(t), \\
& \bar{x}(0)=\bar{x}_{0},
\end{aligned}
$$

where $\bar{w}(t)=L C e(t)+w(t)$.

For system (21), by the impulsive control law $\left\{\tau_{k}, \bar{Q}_{r} x_{r}(t)-\bar{Q} \bar{x}(t)\right\}$ and analysis techniques similar to that in the proof of Theorem 3 , we give the following result without proof.

Theorem 4 Let $P>0,\left\|x_{r}(t)-\bar{x}(t)\right\| \leq \bar{l}\|\bar{x}(t)\|,\|x(t-h)\| \leq \frac{1}{\sqrt{\eta}}\|x(t)\|$ and $\|e(t)\| \leq$ $m\|\bar{x}(t)\|$. Then, the state of system (12) impulsively asymptotically tracks that of the reference system (11) if (20) and the following inequality hold,

$$
0<\eta e^{\frac{\tau}{\eta}}<e^{-\tau\left(1+\lambda_{M}(\bar{E})+\frac{\bar{\mu}^{2}}{\lambda_{m}(P)}\right)}<1
$$

where

$$
\begin{gathered}
\eta=2\left(\lambda_{M}(\bar{F})+\frac{\lambda_{M}(\bar{G})\left(1+\bar{l}^{2}\right)}{\lambda_{m}(P)}\right)>0, \bar{F}=I-2 \bar{Q}+P^{-1} \bar{Q}^{T} P \bar{Q}, \\
\bar{G}=\bar{Q}_{r}^{T} P \bar{Q}_{r}, \bar{E}=A+P^{-1} A^{T} P+B P^{-1} B^{T} P, \bar{\mu}=\|L C\| m+\mu(1+m),
\end{gathered}
$$

Remark 1 In view of Theorem 4, we can see that when system (21) is asymptotically stable, we can apply the estimated system (19) to obtain the state tracking performance index by the impulsive control law $\left\{\tau_{k}, \bar{Q}_{r} x_{r}(t)-\bar{Q} \bar{x}(t)\right\}$. 


\section{Numerical Examples}

In this section, we will present two examples to illustrate our results obtained in Section 3 .

Example 1 Consider the following uncertain time delayed system,

$$
\dot{x}(t)=A_{\Delta} x(t)+B_{1 \Delta}^{T} x\left(t-\tau_{1}(t)\right)+B_{2 \Delta}^{T} x\left(t-\tau_{2}(t)\right)+\omega(t, x),
$$

where

$$
\begin{gathered}
A=\operatorname{diag}(-8.7,-8,-8.9), \omega(t, x)=\left(-\sqrt{x_{2} x_{3}}, \sqrt{x_{1} x_{3}},-\sqrt{x_{1} x_{2}}\right)^{T}, \\
B_{1}=\left(\begin{array}{ccc}
0.05 & 0.25 & 0.05 \\
0.1 & 0.05 & 0.15 \\
0.15 & 0.15 & 0.05
\end{array}\right), B_{2}=\left(\begin{array}{ccc}
0.75 & 0.75 & 0.95 \\
0 & 0.5 & 0.15 \\
0.15 & 0.15 & 0.05
\end{array}\right), \\
F(t)=\left(\begin{array}{ccc}
\sin t & 0 & 0 . \\
0 & 1 & 0 \\
0 & 0 & \cos t
\end{array}\right), \\
x\left(t-\tau_{1}(t)\right)=\left(x_{1}(t-0.2 t), x_{2}(t-0.5 t), x_{3}(t-0.1 t)\right)^{T}, \\
x\left(t-\tau_{2}(t)\right)=\left(x_{1}(t-0.013), x_{2}(t-0.013), x_{3}(t-0.013)\right)^{T} .
\end{gathered}
$$

Obviously

$$
F^{T} F \leq I, l=1, \hat{\tau}_{1}=0.5<1, \hat{\tau}_{2}=0
$$

Let

$$
M=N=L_{1}=L_{2}=Q_{1}=Q_{2}=I,
$$

then we obtain from (4) that

$$
G=\left(\begin{array}{ccc}
-4.09 & 1.33 & 1.54 \\
1.33 & -1.98 & 1.70 \\
1.54 & 1.70 & -3.835
\end{array}\right)<0
$$

and the largest eigenvalue of $G$ is -0.0330 . Thus, system (1) is exponential stable and the convergence rate of exponential stability is $r=0.0165$.

Example 2 Consider the reference system (11) and the tracking system with the following data,

$$
\begin{aligned}
& A_{r}=\left[\begin{array}{cr}
-1.5 & -1.2 \\
2 & -0.2
\end{array}\right], \quad A=\left[\begin{array}{cc}
1.5 & -1 \\
-1 & -2.3
\end{array}\right], B=\left[\begin{array}{cc}
-0.3 & -0.2 \\
0.1 & -0.4
\end{array}\right], C=(1.3,-0.7)^{T}, \\
& x_{r}(0)=(2,-1)^{T}, x(0)=(-2,1)^{T}, w(t)=\left(-\sqrt{\frac{2}{3} x_{1}(t) x_{2}(t)}, \sqrt{\frac{4}{3} x_{1}(t) x_{2}(t)}\right)^{T} .
\end{aligned}
$$

When the state of tracking system is measurable, it is clear to see that we can choose that $\mu=1$. Let $Q_{r}=\operatorname{diag}\left(\frac{1}{2(1+l)}, \frac{1}{2(1+l)}\right), Q=\operatorname{diag}\left(\frac{2}{3}, \frac{2}{3}\right), P=I, \tau=0.01$. Then, we have $\eta=0.7222,-\left(1+\lambda_{M}(E)+\frac{1}{\eta}+\mu^{2}\right) \tau=-0.069932$. Obviously, $\eta=$ $0.7222<e^{-0.069932}=0.9325$. 
Consequently, it follows from Theorem 2 that the state of system (12) asymptotically tracks that of system (11) under the impulsive control law

$$
\left\{\tau_{k}, Q_{r} x_{r}(t)-Q x(t)\right\}
$$

When the state of tracking system is not measurable, we still choose $\mu=1, m=1$. Let $\bar{Q}_{r}=\operatorname{diag}\left(\frac{1}{2(1+\bar{l})}, \frac{1}{2(1+\bar{l})}\right), \bar{Q}=\operatorname{diag}\left(\frac{2}{3}, \frac{2}{3}\right), P=I, \tau=0.01$ and the output feedback gain matrices candidate $L=(1.63,-0.151)^{T}$. Then, we have

$$
\lambda_{M}(J)=-0.0001<0, \eta=0.7222, \bar{\mu}=17.8481,
$$

and $-\left(1+\lambda_{M}(E)+\frac{1}{\eta}+\bar{\mu}^{2}\right) \tau=-0.2384$. It is easy to verify that $\eta=0.7222<$ $e^{-0.2384}=0.7879$.

Consequently, the state of system (2) asymptotically tracks that of system (11) under the impulsive control law

$$
\left\{\tau_{k}, \bar{Q}_{r} x_{r}(t)-\bar{Q} x(t)\right\}
$$

\section{Conclusion}

In this paper, we have investigated exponential stability for a class of control systems with multiple delays and parameter uncertainty. Sufficient conditions are derived on the basis of Lyapunov functional methods and algebraic matrix inequalities. In addition, we proposed novel designed impulsive control laws to achieve the state tracking performance no matter whether the state information is directly available from measurement. Finally, numerical examples are given to illustrate to our results.

\section{References}

1. J. H. Park, S. M. Lee, et al, On exponential stability of bidirectional associative memory neural networks with time-varying delays, Chaos, Solitons and Fractals, 10, 1-9 (2007).

2. J. Cao, J. Liang, et al, Exponential stability of high-order bidirectional associative memory neural networks with time delay, Physica D, 199, 425-436 (2004).

3. J. Liang and J. Cao, Exponential stability of continuous-time and discrete-time bidirectional associative memory networks with delays, Chaos, Solitons and Fractals, 22, 773-785 (2004).

4. E. Yucel and S. Arik, New exponential stability results for delayed neural networks with time varying delays, Physica D, 191, 314-322 (2004).

5. J. H. Park, Robust stabilization for dynamic systems with multiple time-varying delays and nonlinear uncertainties, Journal of Optimization Theory and Applications, 108, 155-174 (2001).

6. X. Li and C. E. De Souza, Criteria for robust stability and stabilization of uncertain linear systems with state-delay, Automatica, 33, 1657-1662 (1997).

7. S. Xu, P. V. Dooren, R. Stefan, et al, Robust stability and stabilization for singular systems with state delay and parameter uncertainty, IEEE Transactions on Automatic Control, 47(7), 1122-1128 (2002).

8. S. Zhou and J. Lam, Robust stabilization of delayed singular systems with linear fractional parametric uncertainties, Circuits Systems Signal Process, 22(6), 579-588 (2003).

9. O. M. Kwon, J. H. Park, Robust stabilization of uncertain systems with delays in control input: a matrix inequality approach, Applied Mathematics and Computation, 172 (2): 10701080 (2006).

10. Cao Y., Sun Y., et al, Delay-dependent robust stabilization of uncertain systems with multiple state delays, IEEE Transactions on Automatic Control, 43, 1608-1612 (1998). 
11. G. A. Rovithakis, Tracking control of multi-input affine nonlinear dynamical systems with unknown nonlinearities using dynamical neural networks, IEEE Transactions on Systems, Man and Cybernetics-Part B, 29, 179-189 (1999).

12. W. E. Schmitendorf, Methods for obtaining robust tracking control laws, Automatic, 23, 675-677 (1987).

13. C. Zhao, K. Ogata and S. Fujii, Adaptive switching control method and its application to tracking control of a robot, in Proceedings of the 1996 IEEE IECON 22nd International Conference on Industrial Electronics, Control, and Instrumentation, 214-219 (1996).

14. J. H. Park. Robust stabilization for dynamic systems with multiple time-varying delays and nonlinear uncertainties. Journal of Optimization Theory and Applications, 108: 155-74 (2001).

15. H. Xu, H. Chen and X. Ding, Impulsive stabilization of cellular neural networks with time delays, Dynamics of Continuous Discrete and Impulsive Systems-Series A- Mathematical Analysis, 41(S1):36-40 (2007).

16. T. Yang , Impulsive control , IEEE Transactions on Automatic Control, 44, 1081-1083 (1999).

17. T. Yang, L.Yang and C. Yang, Impulsive control of Lorenz system, Physica D, 110, 18-24 (1997).

18. R. Luo, Impulsive control and synchronization of a new chaotic system, Acta Physica Sinica, 56, 55-60 (2007).

19. X. Liu and K. L. Teo. Impulsive control of chaotic system. International Journal of Bifurcation and Chaos ,12, 1181-1190 (2002).

20. X. Liu and G. Ballinger, Uniform asymptotic stability of impulsive delay differential equations, Computer and Mathematics with Applications, 41, 903-915 (2001).

21. S. Oucheriah, Robust tracking and model following of uncertain dynamic delay systems by memoryless linear controllers, IEEE Transactions on Automatic Control, 44(7), 1473-1477 (1999).

22. $\mathrm{H}$. $\mathrm{Wu}$, Adaptive robust tracking and model following of uncertain dynamical systems with multiple time delays, IEEE Transaction on Automatic Control, 49(4), 611-616 (2004). 